\title{
CARACTERIZACIÓN SOCIOECONÓMICA DE LOS ESTUDIANTES DE EDUCACIÓN SUPERIOR
}

\section{Introducción ${ }^{1}$}

La reforma de la educación superior de 1981 incorporó nuevos actores institucionales: centros de formación técnica (CFT), institutos profesionales (IP) y amplió el grupo de las universidades (UES) con nuevas instituciones privadas sin aportes estatales, que se agregaron a las instituciones del Estado y a las privadas con aportes financieros de este último. El cuarto de siglo transcurrido bajo esta orgánica significó un incremento explosivo de la matrícula estudiantil de las instituciones autónomas, especialmente a partir de 1996 (Dooner, 2005: 222). Entre los años 1990 y 2004, la matrícula creció un 238\%, impulsada básicamente por la universitaria que subió un 322\%. Las universidades pasaron del 53\% de las matrículas de educación superior en 1990 al 71\% en 2004 y al 80\% en 2006 (PNUD-Mideplan, 2005: 14; CSE, INDICES, 2006).

El aumento en la cobertura de la educación superior (ES) fue estimulado por el incremento del $50 \%$ en los participantes de los procesos Prueba de Admisión Académica-Prueba de Selección Universitaria en la década de los 90 (DEMRE, 2004) hasta el irrefrenable fenómeno expansivo de la admisión 2007 (+32\%) 2 De acuerdo con los datos de la encuesta Casen 2003, entre 1990 y 2003 la cobertura de la educación media subió del 81 al 93\% de la

1 Esta investigación utilizó información de la encuesta de Caracterización Socioeconómica (CASEN) del Ministerio de Planificación. Los resultados del estudio en nada comprometen a dicho ministerio.

2 Producto del movimiento estudiantil de mayo de 2006, el Ministerio de Educación entregó masivamente becas de arancel de la PSU. Casi un 25\% de los inscritos bajo esta modalidad no rindió las pruebas, grupo que hasta ese momento históricamente era en promedio del $5 \%$. Detrás de esta situación se esconden realidades complejas que van desde baja autoestima hasta problemas económicos (Stevens, 2007). 
población en edad escolar, presionando la demanda por educación superior de los grupos sociales más vulnerables, cuya respuesta ha resultado en un avance en la cobertura de ésta para los quintiles ${ }^{3}$ II y III de la población (equivalente al 18\%), quienes habían tenido una participación menor en ese nivel de enseñanza (MIDEPLAN, 2003).

La importancia del tema en estudio reside en que ya no es factible seguir diseñando políticas de educación superior del mismo modo que cuando sólo una elite accedía a la universidad (Leiva, 2005: 42). En la actualidad, es imprescindible producir antecedentes sistemáticos para que el diseño de estas políticas en sus aspectos centrales (orientación de la formación, oferta de carreras, contextualización, soporte para el estudio, financiamiento, entre otros) integre los nuevos actores masivos del sistema, a saber, los estudiantes de los primeros quintiles de la población, pues representan un gran desafío para las políticas públicas (son primera generación en la educación superior), no conocen ni menos manejan los códigos culturales de funcionamiento del "mundo universitario", como tampoco ellos son conocidos cultural y socialmente para la mayor parte de estas instituciones. En segundo lugar, el problema es relevante porque el sistema de educación superior ${ }^{4}$ ha crecido y seguirá creciendo por la integración de estudiantes de los quintiles de menores ingresos económicos (Q1 a Q3) más que por el aumento de la cobertura en los quintiles más pudientes (Q4 y Q5).

En razón de ello, se analizan los principales rasgos socioeconómicos y algunos del ámbito cultural de los estudiantes de educación superior por quintil, según el tipo de institución a la que asisten -CFT, IP y UES-, con la finalidad de que éstas puedan definir políticas para una mejor incorporación y retención de sus estudiantes.

3 El concepto "quintil" se emplea siempre referido a la dimensión socioeconómica autónoma nacional.

4 Ese fue un considerando para impulsar la nueva ley de financiamiento estudiantil universitario, sin distingo de la universidad a la que pertenece, exigiéndose que sea autónoma. 


\section{Expansión de la matrícula de educación superior y retención de los estudiantes}

El fuerte impulso a la demanda por ES del último cuarto de siglo es producto del incremento de la escolaridad promedio de la población (INE, 2004), el cual debería mantener su alza presionado por la obligatoriedad de la educación media que rige desde 2005, sumando a esto el incremento de la eficiencia interna del sistema secundario, egresando más estudiantes de ese nivel (MINEDUC, 2002). Estos factores inciden en el aumento del número de estudiantes habilitados para postular al nivel terciario de la educación. En la última década, la ES creció un 6\% real por año (CSE, 2006) y la tendencia es que continuará en esta senda algunos años más.

La tabla 1 muestra la cobertura por quintil de 1990 a 2003 (CASEN). Ésta creció desde 16,0 a 37,5\% (2,3 veces). Si bien los principales incrementos se producen en los quintiles superiores ${ }^{5}$, se triplica en todo el periodo la cobertura del $40 \%$ de los hogares de menores ingresos: de 4,4 al 14,5\%, en Q1, del 7,8 al 21,3\% en Q2 y de 12,4 al 32,8\% para Q3.

Tabla 1. Cobertura ES por quintil, años 1990 a 2003 (en \%)

\begin{tabular}{l|r|r|r|r|r}
\hline Quintiles & 1990 & 1994 & 1998 & 2000 & 2003 \\
\hline I & 4,4 & 8,0 & 8,7 & 9,4 & 14,5 \\
\hline II & 7,8 & 10,4 & 13,3 & 16,2 & 21,3 \\
\hline III & 12,4 & 17,2 & 23,1 & 28,9 & 32,8 \\
\hline IV & 21, & 33,5 & 38,8 & 43,5 & 46,4 \\
\hline V & 40,2 & 54,2 & 65,5 & 65,6 & 73,7 \\
\hline Cobertura ES & 16 & 23,8 & 29,3 & 31,5 & 37,5 \\
\hline
\end{tabular}

Fuente: PNUD-Mideplan, 2005:48.

La tabla 2 evidencia la mayor semejanza proporcional de estudiantes según quintil entre las universidades (UES) y los institutos profesionales (IP), en especial entre las UES tradicionales con los

5 Para identificar los quintiles se emplea la letra $Q$ acompañada del numeral correspondiente. 
IP. Esto se debe al Fondo Solidario ligado a las UES del Consejo de Rectores, situación que debiese virar en los próximos años por la nueva ley de financiamiento vigente a partir de la admisión 2006.

Tabla 2. Cobertura ES (2003) según institución y quintil (en \%)

\begin{tabular}{l|c|c|c|c|c|c}
\hline & I & II & III & IV & V & Total \\
\hline Universidades & 6,2 & 10,7 & 17,0 & 26,1 & 39,9 & 100 \\
\hline - Tradicionales & 7 & 13,6 & 19,3 & 26,8 & 33,3 & 100 \\
\hline - Privadas & 4,9 & 5,9 & 13,2 & 25,0 & 51,0 & 100 \\
\hline Institutos profesionales & 6,3 & 12,8 & 22,4 & 30,4 & 28,2 & 100 \\
\hline Centros de Formación Técnica & 16 & 22,5 & 26,9 & 17,1 & 17,5 & 100 \\
\hline
\end{tabular}

Fuente: PNUD-Mideplan, 2005: 49.

De no mediar esta nueva ley con características diferentes a las del Fondo Solidario, la expansión del sistema se sustentaba básicamente en el aporte privado, lo que implicaba que estaba próxima a su saturación. Sin embargo, en la nueva ley la condición de "codeudor solidario" de las instituciones puede significar que no se incremente la admisión de los quintiles de menores ingresos, por su mayor riesgo para finalizar la enseñanza, como también que las instituciones se interesen más en promover al alumno que en su aprendizaje. Una fracción de este riesgo se minimiza cuando éstas desarrollan estrategias de apoyo estudiantil a partir del conocimiento de las variables claves y su vinculación con las que son cruciales en su retención.

Las temáticas de la repitencia y la deserción fueron dominantes en los estudios hasta mediados de los 70. La deserción o el abandono anticipado son, conceptualmente, "un caso desviado del objetivo", el cual suele estar precedido por la repitencia, identificada hace tiempo como pródromo de la deserción (Schiefelbein, 1975; Schiefelbein y Simmons, 1981; Schiefelbein y Farrel, 1982: Illanes, 1991). Los estudios en Chile sobre deserción y abandono en la ES son escasos y se orientan esencialmente a aspectos cuantitativos del fenómeno (Himmel, 2002). Se considera que, en promedio, algo menos de la mitad de los alumnos que ingresan a la educación postsecundaria no la alcanza a finalizar exitosamente. En mayor proporción corresponde a deserción durante el 
primer año y atañe a los de menor capital social, cultural y económico (Rodríguez, Donoso y Zunino, 1982; González y Uribe, 2002).

El análisis de la "retención de estudiantes" en Chile se inscribe en un sistema con una tradición de selección de postulantes a las universidades públicas. La instalación de este sistema responde a la hipótesis de que no todos los egresados de la enseñanza secundaria poseen las capacidades para proseguir exitosamente estudios universitarios, debiendo someterse a un proceso selectivo que permita predecir buenos resultados en los estudios universitarios y aumentar la posibilidad de su retención. La incorporación de mayor población estudiantil es clave, dado que el sistema de selección de estudiantes generalizó la creencia que "la selección por mérito" era una materia asociada a la igualdad de oportunidades, ocultando su relación con variables socioeconómicas mediante las oportunidades educativas y éstas con la concepción de equidad social definida por el sistema (González, 2005). En consecuencia, el sistema universitario se desprende de una responsabilidad que le compete en los resultados del estudiante y "privatiza" el fracaso (repitencia y deserción) como responsabilidad propia de éste y no como un proceso en el cual a la institución le corresponde la provisión de los recursos adecuados para que el alumno alcance los aprendizajes esperados, proceso que incluye también políticas compensatorias.

Existen diversas perspectivas teórico-analíticas del fenómeno de la retención. Psicólogos señalan que la decisión de desertar o continuar responde a conductas previas del estudiante: las actitudes sobre la deserción y/o persistencia. Fishbein y Ajzen (1975), asumen la deserción -voluntaria o no-como debilitamiento de las intenciones iniciales y la retención como un fortalecimiento de las mismas. Los resultados empíricos muestran el efecto directo de las aspiraciones en los valores, revelando que las expectativas de éxito estaban explicadas por el autoconcepto académico y la percepción de las dificultades de los estudios (Ethington, 1990). Tanto valores como expectativas influyen en la persistencia en la universidad, validándose la hipótesis de que el rendimiento académico previo influye significativamente 
en el desempeño futuro del estudiante. Sin embargo, como señala Robbins et al. (2004: 263), esta perspectiva experimenta un cambio de significación a partir de la década de los noventa, mediante la integración de enfoques.

Desde la sociología, Spady (1970) sostiene que la deserción/ retención se asocia con la capacidad del estudiante de integrarse a una nueva situación social. El autor señala la gran probabilidad de abandonar los estudios cuando las fuentes de influencia van en sentido opuesto, lo que deriva en un rendimiento académico insatisfactorio, bajo nivel de integración social (factor clave) y, por ende, de satisfacción y compromiso institucional. En la teoría económica se sostiene que cuando los beneficios de los estudios son percibidos como inferiores a los de actividades alternas, los sujetos optan por retirarse. Sin embargo, el estudiante no siempre sigue ese curso racional. En oportunidades los beneficios no son evidentes, como también hay inercia y desfase entre la percepción de un fenómeno y la realidad. Un componente crucial es la percepción de su capacidad/incapacidad para solventar los costos asociados a los estudios. Sin embargo, este factor está mediatizado por otras variables: créditos, becas, entre otros.

La perspectiva organizacional analiza la deserción desde las características institucionales, asumiendo un rol crucial las variables: "calidad de la docencia" y "experiencia del estudiante en el aula", a las que se suman los beneficios de la organización en salud, deportes, cultura, apoyo académico y docente. Estas variables son factibles de ser manejadas por las universidades. El modelo de Tinto (1986, $1987,1975,1997)$ postula que si los beneficios de permanecer en la institución son percibidos como mayores que los costos personales (esfuerzo y dedicación, entre otros), entonces seguirá estudiando. Alternativamente, si reconoce otras actividades como fuente de mayores recompensas el estudiante tenderá a desertar (Véase diagrama 1).

En el tránsito del alumno por la ES diversas variables refuerzan su adaptación: antecedentes familiares, nivel socioeconómico y cultural familiar, valores que sustenta, atributos personales, experiencia 
académica preuniversitaria, los que se combinan en el compromiso inicial con la institución. Tinto considera en la integración académica tanto el rendimiento en este campo como el desarrollo intelectual. Por su parte, la integración social abarca el desarrollo y frecuencia de las interacciones positivas con pares y docentes, y la participación en actividades extracurriculares. Además, establece que la evaluación del compromiso con la meta de graduarse está fuertemente determinada por la integración académica. De igual forma, el compromiso institucional es influenciado por la integración social. Mientras más se consolide el compromiso del estudiante con su graduación y con la institución, simultáneamente será mejor su rendimiento académico e integración social, aumentando la probabilidad de permanecer en la institución. Tinto señala la correspondencia entre habilidad, persistencia y estatus socioeconómico, señalando que no debe sorprender que la persona con más habilidad y mayor nivel socioeconómico sea más propensa a terminar sus estudios (Tinto, 1987: 29-30). Al respecto expresa: "Estudiar, aún en escuelas no selectivas, implica un esfuerzo. Las personas más persistentes van a lograr salir adelante en estos aspectos; sin embargo, esta no es sólo una cualidad individual, es un reflejo también del contexto social y académico" (Tinto, 1987: 42). Es fundamental, entonces, la organización educativa como sistema social y académico en el que se inserta el estudiante. 


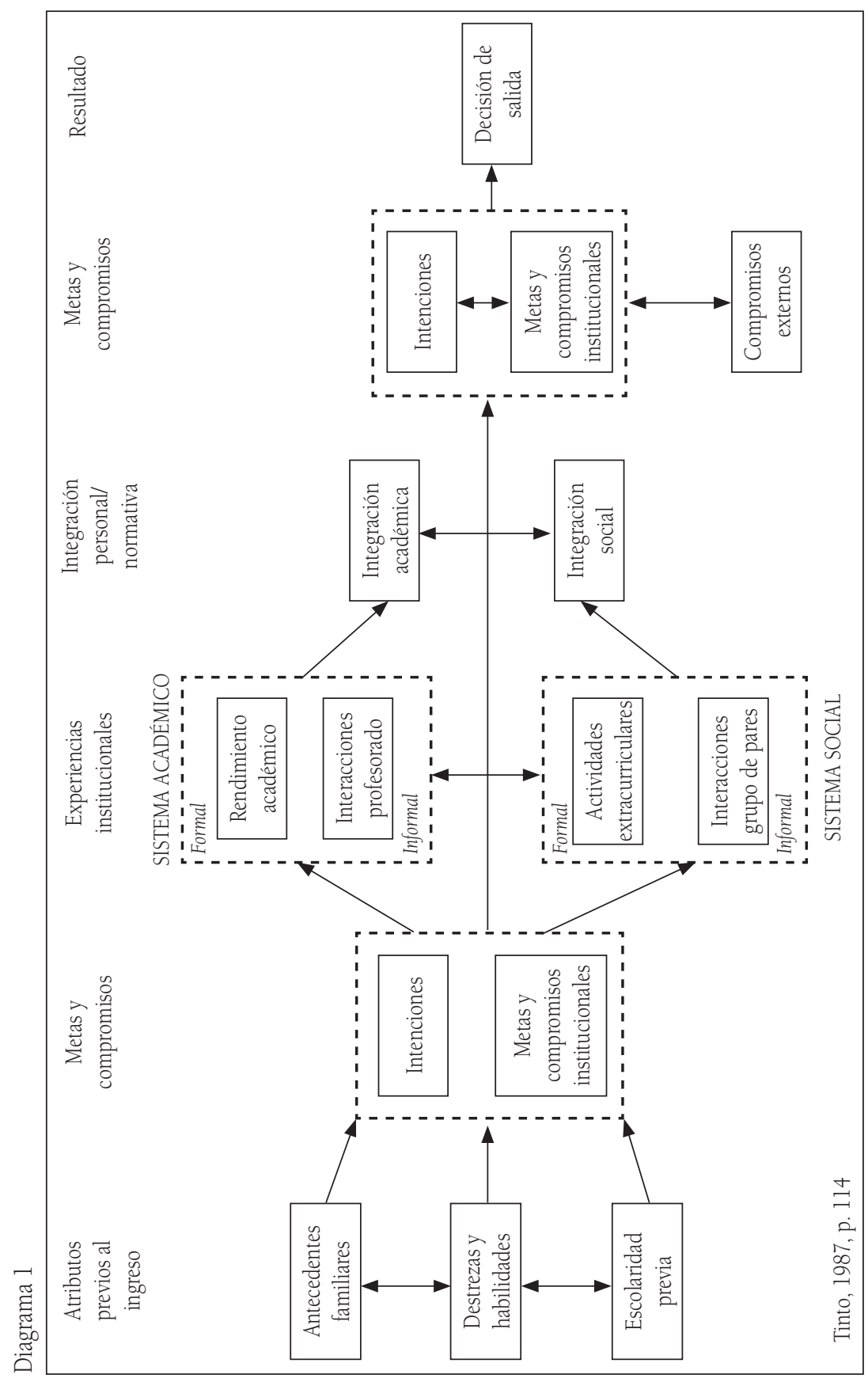


Algunas investigaciones confirman la capacidad predictiva directa de la integración social sobre la deserción en las instituciones de educación (Pascarella y Terenzini, 1980), otras sostienen que se asocia inversamente con la persistencia en los estudios (Pascarella, Smart y Ethington, 1986). Bean (1980, 1983, 1985) asume que la intención de permanecer o retirarse se relaciona con creencias y actitudes, influidas por la institución universitaria (calidad de cursos, programas, docentes y pares), siendo de importancia los factores externos institucionales, pues impactan las actitudes como las decisiones del estudiante. Con posterioridad, identifica aquellos factores no cognitivos: características personales (actitudes, motivaciones, intereses), ambientales y organizacionales tienen peso significativo en la deserción, en particular en la voluntaria. De igual manera, Robinns et al. (2004) atribuyen gran impacto a las variables socioeconómicas en este problema.

La comparación de las dos principales teorías basadas en la persistencia es provista por Robbins et al. (2004), ilustrando las perspectivas de Tinto, Bean y otros (véase tabla 3).

Tabla 3. Modelos de persistencia educativa y de la teoría de la perspectiva motivacional

\begin{tabular}{l|l}
\hline Modelos de persistencia educacional & Teorías motivacionales \\
\hline Influencias contextuales & Motivos como direcciones \\
- Soporte financiero & - Motivación al rendimiento \\
- Tamaño de la institución & Motivos como metas \\
- Selectividad institucional & - Metas académicas \\
Influencia social & - Metas de rendimiento y logro \\
- Soporte social percibido & Motivos como expectativas \\
Contrato social & • Autoeficacia y expectativas de desempeño \\
• Contexto social (integración social, & Autovalor \\
pertenencia social) & - Autoconcepto \\
Contrato académico & \\
• Cometidos referidos al grado & \\
- Cometidos referidos a las instituciones & \\
\hline
\end{tabular}

Robbins et al. (2004: 263).

Por su parte, Pascarella (1986) desarrolla un modelo en función de cinco conjuntos de variables. El primero considera antecedentes 
personales (aptitudes, rendimiento, personalidad, aspiraciones, etnicidad); el segundo características estructurales y organizacionales de la institución (admisión, pares, selectividad); el tercero, el entorno institucional; el cuarto, la interacción con los miembros de la facultad y sus pares, y el quinto, la calidad del esfuerzo desplegado por el estudiante en aprender (Pascarella y Terenzini, 1991: 54-55).

En síntesis, los aspectos esenciales de modelos y enfoques se sustentan en la persistencia, la cual se modela en tres etapas: la inicial, referida a habilidades académicas previas y a factores socioeconómicos del participante que inciden la disposición para continuar ES, como la percepción de sus posibilidades de cumplir; la segunda, en la cual el estudiante "estima" los costos y beneficios de estudiar; la tercera, cuando cursa la ES, comprende factores como las experiencias académicas y sociales y su desempeño académico. Experiencias positivas y un desempeño académico adecuado refuerzan las percepciones de los beneficios económicos y sociales derivados de los estudios. También, los apoyos financieros y una adecuada integración académica influyen en permanecer en la institución, al mantenerse el equilibrio entre el costo de cursar una carrera postsecundaria y el beneficio de obtener un título o grado académico. En cambio, las experiencias negativas producen un desequilibrio, incrementando la probabilidad de la deserción estudiantil.

\section{Los estudiantes de educación superior por quintil}

La hipótesis de trabajo asumida es que la ES chilena ha experimentado un importante proceso de segmentación institucional que correlativamente implica segmentación social. La revisión de la información (tabla 4) establece para la serie considerada (1998, 2000 y 2003) que el número de estudiantes de ES se incrementó significativamente. De 3.984 alumnos en 1998 se pasó a 4.808 en $2000(+20,6 \%)$ y a 6.355 en 2003 , implicando un $32 \%$ de aumento sobre el 2000 y más de 50\% respecto de 1998. 
Tabla 4. Estudiantes de ES según quintil, por años

\begin{tabular}{l|l|r|r|r|r|c|c}
\hline \multirow{2}{*}{ Año } & \multicolumn{6}{|c|}{ Quintil } & \multirow{2}{*}{ Total } \\
\cline { 3 - 8 } & & 1 & 2 & 3 & 4 & 5 & \\
\hline \multirow{2}{*}{2003} & Casos & 626 & 1.006 & 1.308 & 1.608 & 1.807 & 6.355 \\
\hline \multirow{2}{*}{2000} & $\%$ del total & $9,9 \%$ & $15,8 \%$ & $20,6 \%$ & $25,3 \%$ & $28,4 \%$ & $100,0 \%$ \\
\cline { 2 - 8 } & Casos & 532 & 777 & 1.021 & 1.274 & 1.204 & 4.808 \\
\hline \multirow{2}{*}{1998} & $\%$ de total & $11,1 \%$ & $16,2 \%$ & $21,2 \%$ & $26,5 \%$ & $25,0 \%$ & $100,0 \%$ \\
\cline { 2 - 7 } & Casos & 284 & 528 & 767 & 1.092 & 1.313 & 3.984 \\
\hline & $\%$ de total & $7,1 \%$ & $13,3 \%$ & $19,3 \%$ & $27,4 \%$ & $33,0 \%$ & $100,0 \%$ \\
\hline
\end{tabular}

Las diferencias por institución (CFT, IP y UES) expresan que Q4 y Q5 participan activamente, pero con un incremento menor que los primeros tres quintiles, correspondiendo aproximadamente un $7 \%$ entre los años extremos de la serie para todos los niveles. Ello confirma que la expansión considera simultáneamente crecimiento del total de población en edad de estudiar ES.

Los resultados para los CFT confirman que los cambios entre el 2000 y 2003 son menores (+2,7\%). En los IP ello es menos marcado: el incremento va perdiendo significación en tanto se asciende de quintil. Sin embargo, en los IP el alza es significativa entre las últimas dos mediciones $(+8,6 \%)$, algo similar ocurre en las UES. Entre los años extremos el incremento de los primeros tres quintiles es próximo al $100 \%$, mientras que para Q4 y Q5 es de 60 y 40\% (siendo igualmente cuantioso). De algo más del 12\%, entre 1998 a 2000, se sube a casi el $30 \%$ entre 2000 y 2003, ratificando el peso alcanzado por la matrícula universitaria en el sistema de ES del país.

La participación relativa por institución según quintil (tabla 5) afirma lo expuesto. En todos los quintiles los universitarios son fuertes. Los valores van de 61,2\% en Q2 al 79\% en Q5. En los IP los primeros cuatro quintiles dominan ampliamente con valores semejantes $(24,3$ a 26,8\%). En los CFT la participación de los quintiles es menor, nítidamente la de Q5 es exigua: 3,7\%, correspondiendo al 1\% del total. El valor relativo más alto para cada quintil es de Q1 (12,8\%) y el relativo total de Q3 (20\%), sumando los tres primeros quintiles el doble de los otros dos restantes (5,2 versus $2,5 \%$ ). 
En el nivel de los IP la situación es de equilibrio: los primeros tres quintiles alcanzan 11,8\% ante 11,4\% de Q4 y Q5. Finalmente, en UES la relación se invierte respecto de los CFT: los primeros tres quintiles totalizan $29,2 \%$, valor menor que los dos quintiles superiores (suma 10 puntos más: 39,9\%).

Tabla 5. Estudiantes por tipo de institución según quintil (2003)

\begin{tabular}{|c|c|c|c|c|c|c|c|}
\hline & \multicolumn{5}{|c|}{ Quintil } & \multirow[b]{2}{*}{ Total } \\
\hline & & 1 & 2 & 3 & 4 & 5 & \\
\hline CFT & $\%$ por quintil & $12,8 \%$ & $11,9 \%$ & $9,8 \%$ & $5,8 \%$ & $3,7 \%$ & \\
\hline \multirow{2}{*}{ IP } & $\%$ total & $1,3 \%$ & $1,9 \%$ & $2,0 \%$ & $1,5 \%$ & $1,0 \%$ & $7,7 \%$ \\
\hline & $\%$ por quintil & $24,3 \%$ & $26,8 \%$ & $25,4 \%$ & $25,6 \%$ & $17,4 \%$ & \\
\hline \multirow{3}{*}{ UES } & $\%$ total & $2,4 \%$ & $4,2 \%$ & $5,2 \%$ & $6,5 \%$ & $4,9 \%$ & $23,3 \%$ \\
\hline & $\%$ por quintil & $62,9 \%$ & $61,2 \%$ & $64,8 \%$ & $68,6 \%$ & $79,0 \%$ & \\
\hline & $\%$ total & $6,2 \%$ & $9,7 \%$ & $13,3 \%$ & $17,4 \%$ & $22,5 \%$ & $69,0 \%$ \\
\hline Total & $\%$ total & $9,9 \%$ & $15,8 \%$ & $20,6 \%$ & $25,3 \%$ & $28,4 \%$ & $100,0 \%$ \\
\hline
\end{tabular}

Los análisis por tipo de institución y quintil poseen significación estadística por $\mathrm{Chi}^{2}$ (1/8 gl valor $177.955^{* * * 6}$ ). De este modo, las diferencias por quintil e institución confirman rasgos diversos en una población que no puede ser tratada como equivalente. Atendiendo a lo expuesto, se dividieron los participantes según tipo de universidad: las que forman el Consejo de Rectores (UES Cruch) y las que no son subsidiadas UES privadas (UP). En este plano los resultados son relevantes (tabla 6): Q1 está fuertemente presente en las UES Cruch $(48,2 \%)$, valor que va decreciendo en la medida en que se avanza en quintiles, hasta llegar al 40,5\% de Q5. En las UP es diferente: la participación de los primeros dos quintiles es del 14\% en cada uno, elevándose a partir de Q3 al 17,4\%, Q4 a 22,8\% y Q5 al 38,3\%, cifra similar a la registrada por ese quintil en las UES Cruch.

6 Identificación convencional para la significación $(*)$ al 5\%, (**) al l\% y (***) 1 por mil. 
Tabla 6. Estudiantes según dependencia del establecimiento por quintil (2003)

\begin{tabular}{c|l|c|c|c|c|c}
\hline Quintil & & $\begin{array}{c}\text { UES } \\
\text { Cruch }\end{array}$ & $\begin{array}{c}\text { UES } \\
\text { Privadas }\end{array}$ & IP & CFT & Total \\
\hline \multirow{3}{*}{1} & Casos & 302 & 91 & 152 & 80 & 626 \\
\cline { 2 - 7 } & $\%$ quintil & $48,2 \%$ & $14,5 \%$ & $24,3 \%$ & $12,8 \%$ & $100,0 \%$ \\
\cline { 2 - 7 } & $\%$ total & $4,8 \%$ & $1,4 \%$ & $2,4 \%$ & $1,3 \%$ & $9,9 \%$ \\
\hline \multirow{3}{*}{2} & Casos & 478 & 136 & 270 & 120 & 1.006 \\
\cline { 2 - 7 } & $\%$ de quintil & $47,5 \%$ & $13,5 \%$ & $26,8 \%$ & $11,9 \%$ & $100,0 \%$ \\
\cline { 2 - 7 } & $\%$ total & $7,5 \%$ & $2,1 \%$ & $4,2 \%$ & $1,9 \%$ & $15,8 \%$ \\
\hline \multirow{3}{*}{3} & Casos & 617 & 228 & 332 & 128 & 1.308 \\
\cline { 2 - 7 } & $\%$ quintil & $47,2 \%$ & $17,4 \%$ & $25,4 \%$ & $9,8 \%$ & $100,0 \%$ \\
\cline { 2 - 7 } & $\%$ total & $9,7 \%$ & $3,6 \%$ & $5,2 \%$ & $2,0 \%$ & $20,6 \%$ \\
\hline \multirow{3}{*}{4} & Casos & 728 & 366 & 411 & 94 & 1.608 \\
\cline { 2 - 7 } & $\%$ quintil & $45,3 \%$ & $22,8 \%$ & $25,6 \%$ & $5,8 \%$ & $100,0 \%$ \\
\cline { 2 - 7 } & $\%$ total & $11,5 \%$ & $5,8 \%$ & $6,5 \%$ & $1,5 \%$ & $25,3 \%$ \\
\hline \multirow{3}{*}{5} & Casos & 731 & 692 & 314 & 66 & 1.807 \\
\cline { 2 - 7 } & $\%$ quintil & $40,5 \%$ & $38,3 \%$ & $17,4 \%$ & $3,7 \%$ & $100,0 \%$ \\
\cline { 2 - 7 } & $\%$ total & $11,5 \%$ & $10,9 \%$ & $4,9 \%$ & $1,0 \%$ & $28,4 \%$ \\
\hline \multirow{2}{*}{ Total } & Casos & 2.856 & 1.513 & 1.479 & 488 & 6.355 \\
\cline { 2 - 7 } & $\%$ quintil & $44,9 \%$ & $23,8 \%$ & $23,3 \%$ & $7,7 \%$ & $100,0 \%$ \\
\hline
\end{tabular}

Una visión complementaria se obtiene al revisar los resultados por tipo de institución. En las UES Cruch el 51\% de sus estudiantes son de Q4 y Q5, pero en las privadas representa el 70\%. En el caso de los IP, la situación es dicotómicamente similar a las UES públicas: 49\% para los últimos dos quintiles, con la diferencia de que en el grupo de los primeros tres quintiles, Q3 ocupa un lugar más destacado. En los CFT hay una realidad diferente. La participación de Q4 y Q5 se reduce a un tercio $(32,8 \%)$. El $51 \%$ de los participantes corresponde a Q2 y Q3, y al sumarse Q1 el valor se eleva a 66,2\%. En razón de ello, los primeros tres quintiles conforman el grupo mayoritario de estas instituciones (estadísticamente significativos al 1/000).

La revisión de los estudiantes de las UES por quintil ratifica lo expuesto. Los de menores ingresos asisten masivamente a las UES Cruch: 81,5\% de Q1, 80\% de Q2, hasta llegar al 51,4\% de Q5. En consecuencia, menos del 20\% de los primeros dos quintiles está en las UP, hasta llegar prácticamente al 48\% de Q5 que está en las privadas. Proporcionalmente, por cada alumno de Q1 que está en una UP hay 4,4 en una pública, 
situación que respecto de Q2 es de 1 a 3,9, en Q3 de 1 a 3, 1 estudiantes, en Q4 de 1 a 2,1 y, finalmente, en Q5 es casi equivalente de 1 estudiante en universidad privada por 1,05 en una pública.

En síntesis, el análisis establece dos aspectos centrales: uno es la sistemática y gran concentración de estudiantes del nivel universitario; el segundo aspecto tiene una doble faz: (i) cualquiera que sea el quintil de pertenencia, la mayoría de los estudiantes participan de las UES, (ii) hay tendencias relevantes por tipo de institución: en los CFT predominan los primeros quintiles, en los IP la situación es más equilibrada, con más presencia de los quintiles intermedios, y en las UES el escenario mayoritario corresponde a Q4 y Q5, lo que, en el caso de las UP, es todavía más concentrado. Si bien esto se refiere a tendencias, se trata de relaciones estadísticamente significativas.

\subsection{Los estudiantes por género}

Producto de sus mejores rendimientos históricos en la enseñanza secundaria (MINEDUC, 2004), las mujeres representan al menos el $50 \%$ de la matrícula de ES. Ello se refleja en el estudio, no hay diferencias por género pero sí por quintil.

Tabla 7. Estudiantes por género y quintil (2003)

\begin{tabular}{l|l|c|c|c|c|c|c}
\hline \multirow{2}{*}{ Género } & \multicolumn{5}{|c|}{ Quintil } & \multirow{2}{*}{ Total } \\
\cline { 3 - 8 } \multicolumn{2}{l|}{} & 1 & 2 & 3 & 4 & 5 & \\
\hline \multirow{2}{*}{ Hombre } & Casos & 256 & 445 & 630 & 870 & 976 & 3.177 \\
\hline \multirow{3}{*}{ Mujer } & $\%$ por quintil & $40,9 \%$ & $44,2 \%$ & $48,2 \%$ & $54,1 \%$ & $54,0 \%$ & $50,0 \%$ \\
\hline \multirow{3}{*}{ Total } & $\%$ total & $4,0 \%$ & $7,0 \%$ & $9,9 \%$ & $13,7 \%$ & $15,4 \%$ & $50,0 \%$ \\
\cline { 2 - 8 } & Casos & 370 & 561 & 678 & 738 & 831 & 3.178 \\
\cline { 2 - 8 } & $\%$ por quintil & $59,1 \%$ & $55,8 \%$ & $51,8 \%$ & $45,9 \%$ & $46,0 \%$ & $50,0 \%$ \\
\hline \multirow{3}{*}{} & $\%$ total & $5,8 \%$ & $8,8 \%$ & $10,7 \%$ & $11,6 \%$ & $13,1 \%$ & $50,0 \%$ \\
\cline { 2 - 8 } & Casos & 626 & 1.006 & 1.308 & 1.608 & 1.807 & 6.355 \\
\cline { 2 - 8 } & $\%$ por sexo & $9,9 \%$ & $15,8 \%$ & $20,6 \%$ & $25,3 \%$ & $28,4 \%$ & $100,0 \%$ \\
\hline & $\%$ por quintil & $100,0 \%$ & $100,0 \%$ & $100,0 \%$ & $100,0 \%$ & $100,0 \%$ & $100,0 \%$ \\
\hline
\end{tabular}

Las mujeres son mayoría en los primeros tres quintiles. Con fuerza en Q1 (59\%) hasta un mayoría relativa en Q3 (52\%). Los hombres dominan en los dos quintiles superiores, con una diferencia 
de ocho puntos porcentuales. Esta situación tiene significación estadística, teórica, práctica y metodológica $\left(\mathrm{Chi}^{2}, 1 / 4 \mathrm{gl}, 58.36^{* * *}\right)$. En lo teórico implica que la población de ES con mayores dificultades socioeconómicas son las mujeres. Igualmente, la consolidación de su participación de Q5 en el 46\% es destacable, cuyo origen está por conocerse. En lo práctico, el incremento de estudiantes de los primeros quintiles demanda el soporte educacional y social para que se traduzca en resultados positivos: políticas de orientación sexual y de la natalidad, apoyo a la maternidad, entre otras medidas. En materia de políticas educativas, se requiere analizar los estilos de aprendizaje y los sesgos de los resultados de la PSU según género, e identificar estrategias para fortalecer las áreas más débiles.

En asuntos metodológicos, son importantes las diferencias por género en lo que respecta a los primeros tres quintiles, lo que implica que los análisis deben considerar esta condición.

La tabla 8 presenta la distribución por género y tipo de institución. Si bien la tendencia es mayor presencia femenina en los CFT y UES (51,5\%), la diferencia se invierte en los IP con magnitud de 10 puntos (45,3\% mujeres y 54,7\% hombres), (Chi $\left.{ }^{2} 17,193 * * *\right)$, respondiendo probablemente a la orientación de los programas académicos de esas instituciones.

Tabla 8. Estudiantes por género según tipo de institución (2003)

\begin{tabular}{l|l|c|c|c|c}
\hline \multirow{2}{*}{ Sexo } & & \multicolumn{3}{|c|}{ Tipo } & \multirow{2}{*}{ Total } \\
\cline { 3 - 5 } & & CFT & IP & UES & \\
\hline Hombre & Casos & 239 & 809 & 2.131 & 3.179 \\
\hline & $\%$ por sexo & $7,5 \%$ & $25,4 \%$ & $67,0 \%$ & $100,0 \%$ \\
\hline \multirow{3}{*}{ Mujer } & $\%$ por tipo & $48,6 \%$ & $54,7 \%$ & $48,5 \%$ & $50,0 \%$ \\
\hline & $\%$ total & $3,8 \%$ & $12,7 \%$ & $33,5 \%$ & $50,0 \%$ \\
\cline { 2 - 5 } & Casos & 253 & 670 & 2.259 & 3.182 \\
\cline { 2 - 5 } & $\%$ por sexo & $8,0 \%$ & $21,1 \%$ & $71,0 \%$ & $100,0 \%$ \\
\cline { 2 - 5 } & $\%$ por tipo & $51,4 \%$ & $45,3 \%$ & $51,5 \%$ & $50,0 \%$ \\
\hline \multirow{2}{*}{ Total } & $\%$ total & $4,0 \%$ & $10,5 \%$ & $35,5 \%$ & $50,0 \%$ \\
\cline { 2 - 5 } & Casos & 492 & 1.479 & 4.390 & 6.361 \\
\hline & \% por sexo & $7,7 \%$ & $23,3 \%$ & $69,0 \%$ & $100,0 \%$ \\
\hline
\end{tabular}


La revisión por género y quintil, según institución, confirma lo establecido, adicionando elementos significativos ( $\mathrm{Chi}^{2}$ 92.4*** hombres y $97,1^{* * *}$ mujeres con $1 / 8$ gl). Primero, la participación relativa masculina en Q4 y Q5 es del 58\%, y femenina 49,3\%. Proporcionalmente, los hombres se agrupan en los quintiles superiores. Ello se explica por la participación de los primeros dos quintiles, que equivale al 22,1\% de los hombres y $29,3 \%$ de mujeres.

Las mujeres aumentan su presencia en las UES en cuanto se asciende en quintil. De 0,7 a su favor en Q1, se pasa a 4,9\% en Q5. Para las mujeres, mientras mayor es proporcionalmente su quintil de pertenencia, mayor su participación relativa en las UES. De esta manera, 62,5\% de hombres de Q1 está en la universidad y de Q5 el $76,7 \%$ (en los quintiles intermedios la progresión es variable, producto de la influencia de los que están en IP que también suben). En el caso de las mujeres, la situación es: Q1 63,2\%, Q5, 81,6\%.

En los IP la presencia masculina es superior desde Q2 en adelante, decreciendo fuertemente en Q5, siendo más aguda en las mujeres (posiblemente debido a su participación en las UES). En los CFT hay predominio relativo femenino en los dos primeros quintiles, en los tres superiores hay una leve ventaja porcentual de los hombres.

Finalmente, según tipo de UES, la participación relativa de ambos géneros en el total es muy similar, y la de las mujeres en las UES públicas cae de manera abrupta cuando se sube de quintil: de 81,6\% en Q1 a 49,4\% en Q5. Lo opuesto se registra en las privadas para ambos géneros, aunque es más claro en las mujeres, pues sube de $18,4 \%$ en Q1 al 50,6\% en Q5.

Para efectos del estudio, el análisis por género es importante. Las mujeres forman el grupo más numeroso de los primeros tres quintiles. En los dos superiores la situación responde a los hombres. Sin embargo, la participación relativa de ellas en las UES sigue el sentido de los quintiles. Esta realidad no tiene el mismo sentido en los IP, mientras que para los CFT las mujeres son dominantes en los primeros quintiles y los hombres en los últimos. 


\subsection{Caracterización de los estudiantes por edad}

La variable edad es relevante cuando se asocia al costo oportunidad de los estudios. Tanto más se progresa en edad se eleva también el costo oportunidad de estudiar, el que, además, varía según el quintil de pertenencia del estudiante: a menor quintil y mayor edad el costo oportunidad de estudiar es superior. La revisión de los resultados siguientes (tabla 9) es consistente con la hipótesis precedente. Los estudiantes de Q1 son más jóvenes: 39,3\% tiene hasta 20 años, valor que se reduce en cuanto se asciende de quintil, llegando a $26,7 \%$ en Q5. Esta situación se repite con menor rango para el tramo de edad siguiente ( 21 y 22 años). Ello se equilibra en el intervalo de edad superior (23 a 24), iniciando su reversión hasta llegar a una situación opuesta: en los estudiantes de más de 30 años (7,1\% del total) un tercio pertenece a Q5 y casi otro tanto a Q4, los de Q1 son casi 1/6 menos que $\mathrm{Q} 5$, ratificando que el costo oportunidad por edad incide en que los primeros quintiles tengan menor edad $\left(\mathrm{Chi}^{2}\right.$ de $89,17^{* * *}$ $1 / 16 \mathrm{gl})$.

Tabla 9. Estudiantes por quintil y rangos de edad (2003)

\begin{tabular}{|c|c|c|c|c|c|c|c|}
\hline & \multirow[b]{2}{*}{ Quintil } & \multicolumn{5}{|c|}{ Edad } & \multirow[b]{2}{*}{ Total } \\
\hline & & \multirow{2}{*}{$\begin{array}{l}\text { hasta } \\
20 \text { años } \\
246\end{array}$} & \multirow{2}{*}{$\frac{21 \text { a } 22}{170}$} & \multirow{2}{*}{$\frac{23 \text { a } 24}{104}$} & \multirow{2}{*}{$\frac{25 \text { a } 30}{79}$} & \multirow{2}{*}{$\begin{array}{r}31 y+ \\
27\end{array}$} & \\
\hline 1 & Casos & & & & & & 626 \\
\hline & $\%$ de quintil & $39,3 \%$ & $27,2 \%$ & $16,6 \%$ & $12,6 \%$ & $4,3 \%$ & $100,0 \%$ \\
\hline \multirow{3}{*}{2} & $\%$ del total & $3,9 \%$ & $2,7 \%$ & $1,6 \%$ & $1,2 \%$ &, $4 \%$ & $9,9 \%$ \\
\hline & Casos & 367 & 274 & 172 & 147 & 46 & 1.006 \\
\hline & $\%$ de quintil & $36,5 \%$ & $27,2 \%$ & $17,1 \%$ & $14,6 \%$ & $4,6 \%$ & $100,0 \%$ \\
\hline \multirow{3}{*}{3} & $\%$ del total & $5,8 \%$ & $4,3 \%$ & $2,7 \%$ & $2,3 \%$ &, $7 \%$ & $15,8 \%$ \\
\hline & Casos & 394 & 351 & 218 & 249 & 96 & 1.308 \\
\hline & $\%$ de quintil & $30,1 \%$ & $26,8 \%$ & $16,7 \%$ & $19,0 \%$ & $7,3 \%$ & $100,0 \%$ \\
\hline \multirow{3}{*}{4} & $\%$ del total & $6,2 \%$ & $5,5 \%$ & $3,4 \%$ & $3,9 \%$ & $1,5 \%$ & $20,6 \%$ \\
\hline & Casos & 464 & 427 & 288 & 295 & 134 & 1.608 \\
\hline & $\%$ de quintil & $28,9 \%$ & $26,6 \%$ & $17,9 \%$ & $18,3 \%$ & $8,3 \%$ & $100,0 \%$ \\
\hline \multirow{3}{*}{5} & $\%$ del total & $7,3 \%$ & $6,7 \%$ & $4,5 \%$ & $4,6 \%$ & $2,1 \%$ & $25,3 \%$ \\
\hline & Casos & 483 & 456 & 344 & 375 & 149 & 1.807 \\
\hline & $\%$ de quintil & $26,7 \%$ & $25,2 \%$ & $19,0 \%$ & $20,8 \%$ & $8,2 \%$ & $100,0 \%$ \\
\hline \multirow{3}{*}{ Total } & $\%$ del total & $7,6 \%$ & $7,2 \%$ & $5,4 \%$ & $5,9 \%$ & $2,3 \%$ & $28,4 \%$ \\
\hline & Casos & 1.954 & 1.678 & 1.126 & 1.145 & 452 & 6.355 \\
\hline & $\%$ de quintil & $30,7 \%$ & $26,4 \%$ & $17,7 \%$ & $18,0 \%$ & $7,1 \%$ & $100,0 \%$ \\
\hline
\end{tabular}


La información según género es estadísticamente significativa (hombres $\mathrm{Chi}^{2}$ de $34,98^{* *}$, mujeres $\mathrm{Chi}^{2} 74,99^{* * *}$ ). Los hombres de los primeros tres quintiles son más jóvenes que los de Q4 y Q5, confirmando que el costo oportunidad de estudiar en esta población es superior y se asocia a edades próximas al ciclo regular de estudios. Las mujeres de los primeros quintiles son relativamente más que los hombres, siguiendo la declinación ya registrada en la medida en que se asciende de quintil. Los hombres de Q4 y Q5 son más que las mujeres de estos quintiles, sin embargo, se distribuyen de forma similar, recordando que más del 55\% de los estudiantes de todas las edades pertenecen a los dos quintiles superiores, cifra que llega al $60 \%$ en las edades/quintiles superiores.

La información por edad y tipo de institución revalida que proporcionalmente en los CFT están los más jóvenes. El 35,2\% de hasta 20 años, frente al 32,5\% en los IP y el 29,7\% en las UES. Debe recordarse que el grupo de las UES es el más numeroso. Sin embargo, paradójicamente en los CFT está el grupo de mayor edad relativo (31 años y más). Si bien sabemos que representan el 7,1\% del total de participantes, en los CFT su peso sube a $8,9 \%$, bajando en los IP al $5,7 \%$ y acercándose al promedio en las UES. Ello permitiría pensar que el racional de este grupo es diferente en cierto grado del de edades precedentes.

Finalmente, los resultados de los estudiantes de las UES públicas y privadas muestran varios aspectos referidos previamente. En el grupo más joven existe una fuerte presencia de estudiantes de UP de Q5 (44,6\%). Si bien ello se repite en todos los quintiles, incluso con participaciones superiores, es un aspecto que es transversal al sistema, siempre en referencia a Q5. Respecto de Q4, la participación relativa comparada por tramo de edad y UES es muy similar, habiendo cambios menores. Esa situación se invierte en Q3 con diferencias significativas hacia las UES públicas, lo que también se extiende a los participantes de Q2. Es respecto de Q1 donde surge un resultado algo distinto. Aunque en el grupo más joven predominan estudiantes de UES públicas, en el tramo siguiente de edad -21 a 22 años- hay 
muy pocos estudiantes de este quintil en UP ( $2,7 \%$ del grupo etario), mientras que en las UES públicas es casi del 11\%. Esta situación vuelve a sus diferencias convencionales en los tramos siguientes de edad.

En síntesis, la edad es un factor relevante en la caracterización de la población, entendiendo que, en su gran mayoría, se concentra en valores menores de 25 años. Los estudiantes de los primeros quintiles tienen menor edad, como también es cuantitativamente menor el grupo de estudiantes de mayor edad que pertenece a los primeros quintiles. Ello ratifica que existe un costo/oportunidad superior para los grupos menos favorecidos económicamente y que se incrementa con la edad. El análisis por institución muestra que, proporcionalmente, en los CFT está la gente más joven; sin embargo, paradójicamente, en estas instituciones también están -en términos relativos- los de mayor edad. Finalmente destaca para el Q5 su fuerte participación en las UES privadas; si bien no supera en términos absolutos al grupo de las públicas, proporcionalmente representa un valor muy superior de estudiantes en todas las edades.

\section{Los estudiantes por tipo de institución: centros de formación técnica, institutos profesionales y universidades}

\subsection{Rasgos comunes}

Los resultados de la serie de años $(1998,2000,2003)$ caracterizan a los estudiantes del sistema de ES con ciertos rasgos compartidos o comunes, producto de la selectividad del sistema escolar desde sus inicios, provocando que presenten valores semejantes en variables claves. Esta sección se orienta a analizar los elementos compartidos de mayor significación.

El principal rasgo común del sistema de ES chileno es que exige a sus alumnos dedicación exclusiva al estudio o bien genera incompatibilidad importante con la actividad laboral simultánea. Condición cumplida al menos por el 80\% de sus integrantes. De 
esta manera el sistema "modela" una tipología de estudiante que se impone. Aunque este fenómeno no es nuevo, sí es apreciable, pues la "dedicación exclusiva al estudio" demanda un soporte financiero y de calidad de vida razonable. Este rasgo sistémico implica que el estudiante se adapte "con carácter casi excluyente de toda otra actividad", en vez de que el sistema se asimile al estudiante. Esta característica de la ES ha funcionado como auto filtro para seleccionar estudiantes (Stevens, 2007), fortaleciendo la semejanza más allá de lo que inicialmente se pensaba en variables claves de la población estudiantil.

Cuando se analiza la incorporación de nuevos estudiantes a la ES a partir de la categorización de los quintiles como criterios estanco, asentadas en diferencias absolutas, se puede generar una visión distorsionada de ellos, creyendo que, por fin, los más pobres se incorporan masivamente a ella, pues todos los quintiles han duplicado su participación. Los avances en este campo son importantes, como igualmente lo es comprender que a la ES no llega masivamente la población indigente (-2\% pertenece a ese grupo) y accede una fracción menor de pobres (6\%), de forma que, esencialmente, la población estudiantil -con algunos bemoles analizados más adelante- está compuesta masivamente por estudiantes no pobres (92\%). Esta última categorización es amplia, se sustenta en parámetros menos variables que la estructura de quintiles, expresando que la satisfacción de las necesidades esenciales es en términos absolutos y no relativos como establece el análisis por quintiles ${ }^{7}$. Los estudiantes de ES pertenecen mayoritariamente a los últimos dos quintiles, con presencia creciente de personas de los primeros tres.

La condición de estudiante de dedicación exclusiva demanda un soporte básico de calidad de vida de la familia o del grupo de referencia del participante, que deriva en semejanzas importantes en algunas

7 En el análisis se emplean ambos modos de clasificación, aunque, de preferencia, se utiliza el de quintiles dada su generalización comparativa y su uso para asignar beneficios a la población. 
dimensiones, a saber: equipamiento básico del hogar (acceso a servicios básicos, agua caliente, entre otros), condiciones de habitabilidad de la vivienda, condiciones de uso y propiedad de la misma, provocando que no sean relevantes en término de grandes diferencias, pues, salvo excepciones, el acceso es bastante universal a una situación inicial que permite la dedicación al estudio. También, ello implica postergación de bienestar o esfuerzo del grupo familiar para generar y mantener este soporte, tema que se debate al final de la sección.

Complementariamente, los datos del acceso a los servicios de salud, si bien presentan algunas diferencias por quintil, no muestran desamparo de unos frente a cobertura de otros. Hay algunas situaciones preocupantes, referidas fundamentalmente a cobertura de atención de salud de las mujeres, generalizadamente asumida por el sector público, lo que muestra las políticas del país en este ámbito y las precauciones del sistema privado en la materia.

De igual forma, esta situación deriva en que la composición del estudiantado según edad y estado civil sea muy similar: generalizadamente solteros y sin hijos. También lo es en referencia a su situación laboral. En su gran mayoría, los estudiantes no trabajan remuneradamente (80\%) -aunque hay matices menores-; muy pocos buscan empleo sistemáticamente (-4\%); no más del 20\% tiene alguna actividad remunerada de cierta estabilidad, con mayor fuerza en los CFT (27\%), luego en los IP (23\%) y, finalmente, en las UES (20\%). La pertenencia a los primeros quintiles no implica tener más trabajo ni tampoco buscarlo en proporción mayor que en otros quintiles.

A partir de los resultados, si bien se sostiene que los estudiantes, según tipo de institución, responden a segmentos sociales diferentes, debe comprenderse que las discrepancias se fundan en la estructura de quintiles. En los CFT se concentran proporcionalmente más estudiantes de los primeros tres quintiles que en los IP y las UES. También se manifiesta en que, socioeconómicamente, la población de los IP es más semejante a la de las UES públicas, no así de las privadas. 
En materia de género, los IP están sesgados hacia los estudiantes hombres, fenómeno que no se da con esa fuerza en los otros segmentos de la ES. Finalmente, las diferencias por quintil entre las UES públicas y privadas dan como resultado que, en estas últimas, se integran en mayor medida (relativa) quienes pertenecen a Q5, respondiendo ello a fenómenos de orientación de mercado, precio de las carreras, exigencias de admisión, sistema de becas de sustento y otros factores asociados.

En este marco es relevante destacar que la población más pobre es significativamente femenina, cualquiera sea la categoría empleada para definirla (pobreza o quintil). Ello genera un conjunto de consecuencias importantes para las personas, sus familias, las instituciones y la sociedad, que deberían atenderse con mayor dedicación.

4.2. Los estudiantes de los centros de formación técnica (CFT) El grupo muestral lo conforman 492 alumnos de CFT que, mayoritariamente, pertenecen a los primeros tres quintiles. Sin embargo, en la medida en que aumenta la edad de los alumnos también lo hace la participación de los quintiles superiores. Esto se debe a la relación costo/oportunidad que conlleva estudiar y su vínculo con la variable edad.

Estos estudiantes se diferencian de los otros niveles de ES porque son más jóvenes (el 62\% tiene hasta 22 años) y, también, porque están los de mayor edad relativa del sistema (9\% tiene 31 años y más), estos últimos masivamente casados o separados, perteneciendo de preferencia a los dos quintiles de mayor holgura socioeconómica.

Tabla 10. Edad por quintil-CFT

\begin{tabular}{l|l|c|c|c|c|c|c}
\hline \multirow{2}{*}{ Edad } & & \multicolumn{5}{|c|}{ Quintil } & \multirow{2}{*}{ Total } \\
\cline { 3 - 8 } & & 1 & 2 & 3 & 4 & 5 & \\
\hline Hasta 20 años & Casos & 32 & 57 & 41 & 26 & 15 & 171 \\
\hline \multirow{2}{*}{21 a 22 } & $\%$ quintil & $40,0 \%$ & $47,5 \%$ & $32,0 \%$ & $27,7 \%$ & $22,7 \%$ & $35,0 \%$ \\
\hline & $\%$ total & $6,6 \%$ & $11,7 \%$ & $8,4 \%$ & $5,3 \%$ & $3,1 \%$ & $35,0 \%$ \\
\cline { 2 - 8 } & Casos & 29 & 28 & 37 & 26 & 14 & 134 \\
\cline { 2 - 8 } & $\%$ quintil & $36,3 \%$ & $23,3 \%$ & $28,9 \%$ & $27,7 \%$ & $21,2 \%$ & $27,5 \%$ \\
\hline
\end{tabular}




\begin{tabular}{l|l|c|c|c|c|c|c}
\hline \multirow{2}{*}{23 a 24} & $\%$ total & $5,9 \%$ & $5,7 \%$ & $7,6 \%$ & $5,3 \%$ & $2,9 \%$ & $27,5 \%$ \\
\cline { 2 - 8 } & Casos & 9 & 18 & 12 & 17 & 16 & 72 \\
\cline { 2 - 8 } & $\%$ quintil & $11,3 \%$ & $15,0 \%$ & $9,4 \%$ & $18,1 \%$ & $24,2 \%$ & $14,8 \%$ \\
\hline \multirow{2}{*}{25 a 30} & $\%$ total & $1,8 \%$ & $3,7 \%$ & $2,5 \%$ & $3,5 \%$ & $3,3 \%$ & $14,8 \%$ \\
\cline { 2 - 8 } & Casos & 6 & 12 & 22 & 15 & 12 & 67 \\
\cline { 2 - 8 } & $\%$ quintil & $7,5 \%$ & $10,0 \%$ & $17,2 \%$ & $16,0 \%$ & $18,2 \%$ & $13,7 \%$ \\
\hline \multirow{3}{*}{31 y más años } & $\%$ total & $1,2 \%$ & $2,5 \%$ & $4,5 \%$ & $3,1 \%$ & $2,5 \%$ & $13,7 \%$ \\
\cline { 2 - 8 } & Casos & 4 & 5 & 16 & 10 & 9 & 44 \\
\cline { 2 - 8 } & $\%$ quintil & $5,0 \%$ & $4,2 \%$ & $12,5 \%$ & $10,6 \%$ & $13,6 \%$ & $9,0 \%$ \\
\hline \multirow{2}{*}{ Total } & $\%$ total &, $8 \%$ & $1,0 \%$ & $3,3 \%$ & $2,0 \%$ & $1,8 \%$ & $9,0 \%$ \\
\hline & Casos & 80 & 120 & 128 & 94 & 66 & 488 \\
\hline & $\%$ total & $16,4 \%$ & $24,6 \%$ & $26,2 \%$ & $19,3 \%$ & $13,5 \%$ & $100,0 \%$ \\
\hline
\end{tabular}

Analizados detalladamente, los estudiantes indigentes y pobres no presentan diferencias importantes en variables como acceso a computador, sistemas públicos de salud, uso de la vivienda, entre otras. Incluso, la población indígena no es dominante en estos grupos. Es más, algunas diferencias les discriminan negativamente. Por ejemplo, los indigentes casi no tienen acceso a becas de apoyo y "los pobres no indigentes" tampoco. Adicionalmente, las becas estatales de colegiatura son asignadas masivamente a los no pobres y sólo a algunos pobres, mostrando que esta situación no es garantía para la obtención de esos recursos. El 82\% de los participantes totales no recibe apoyo, lo que puede deberse al sistema de asignación de beneficios: si se basa sólo en criterios de rendimiento académico es muy posible que la exclusión social esté presente.

En materia de empleo -comparados con los de IP y UEStrabajan en mayor proporción (27\%), a pesar de que quienes vienen de los primeros quintiles constituyen este grupo de manera importante. Sin embargo, en Q1 y Q2 el peso de los que no trabajan es superior al del grupo total. Según género, los hombres trabajan en mayor proporción; en el grupo de los CFT, en general, el empleo es frágil: no más del $41 \%$ tiene contrato indefinido. 
Tabla 11. Trabajo por quintil- CFT

\begin{tabular}{l|l|c|c|c|c|c|c}
\hline \multirow{2}{*}{} & & \multicolumn{7}{|c|}{ Quintil } & \multirow{2}{*}{ Total } \\
\cline { 3 - 8 } & & 1 & 2 & 3 & 4 & 5 & \\
\hline \multirow{2}{*}{ Sí } & Casos & 9 & 17 & 50 & 32 & 24 & 132 \\
\hline & $\%$ itrabajó? & $6,8 \%$ & $12,9 \%$ & $37,9 \%$ & $24,2 \%$ & $18,2 \%$ & $100,0 \%$ \\
\hline \multirow{3}{*}{ No } & $\%$ quintil & $11,3 \%$ & $14,2 \%$ & $39,1 \%$ & $34,0 \%$ & $36,4 \%$ & $27,0 \%$ \\
\hline \multirow{3}{*}{ Total } & $\%$ total & $1,8 \%$ & $3,5 \%$ & $10,2 \%$ & $6,6 \%$ & $4,9 \%$ & $27,0 \%$ \\
\cline { 2 - 8 } & Casos & 71 & 103 & 78 & 62 & 42 & 356 \\
\cline { 2 - 8 } & $\%$ itrabajó? & $19,9 \%$ & $28,9 \%$ & $21,9 \%$ & $17,4 \%$ & $11,8 \%$ & $100,0 \%$ \\
\cline { 2 - 8 } & $\%$ quintil & $88,8 \%$ & $85,8 \%$ & $60,9 \%$ & $66,0 \%$ & $63,6 \%$ & $73,0 \%$ \\
\hline & $\%$ total & $14,5 \%$ & $21,1 \%$ & $16,0 \%$ & $12,7 \%$ & $8,6 \%$ & $73,0 \%$ \\
\cline { 2 - 8 } & Casos & 80 & 120 & 128 & 94 & 66 & 488 \\
\hline & $\%$ itrabajó? & $16,4 \%$ & $24,6 \%$ & $26,2 \%$ & $19,3 \%$ & $13,5 \%$ & $100,0 \%$ \\
\hline
\end{tabular}

Las cifras de la tabla anterior muestran (significación estadística) que para Q1 y Q2 el peso de los que no trabajan es superior al valor de la distribución total (+ 16 y 13 respectivamente). Son los de Q3 quienes poseen mejores tasas de trabajo. Ello no se compadece con la idea de que los sujetos de los primeros quintiles tienen mayores requerimientos de soporte financiero. Las explicaciones de esto son variadas. Una provendría de la cobertura de becas que, como se señala, no siempre están focalizadas en los primeros quintiles; otra viene de la estructura de salarios, según el capital social y educacional de los estudiantes. Ello avalaría que los alumnos de los primeros quintiles accedan a empleos de menor retribución económica, justificando la necesidad de estudiar precisamente para mejorar las posibilidades de obtener un trabajo de mejor calidad. Sin embargo, es un punto que debemos considerar.

Respecto del apoyo de becas: 26 estudiantes (5,3\%) tienen la Beca Presidente de la República, 13 del primer quintil, cinco del segundo, siete del tercero y uno del cuarto. Otros 29 disponen de otras becas estatales, representando el 5,8\% (seis de Q1, 10 de Q2 y ocho de Q3). Se suman otros 25 participantes con becas no estatales $(5,1 \%)$, distribuidos siete casos en cada uno de los primeros tres quintiles. Por lo menos el $82 \%$ no recibe apoyo financiero directo e indirecto de entidades públicas o privadas. Desde el punto de vista de género, la distribución de las becas es homogénea. 
Finalmente, la distribución espacial de los estudiantes de CFT refleja la estructura de oportunidades del país. Si bien la relación quintil por región que habita no permite una interpretación exhaustiva de los casos, por el bajo número registrado en algunas regiones ${ }^{8}$, existe plena concordancia entre los estándares nacionales de distribución por quintiles socioeconómicos y los resultados logrados. A modo de ejemplo, para la Región de Valparaíso, el 35\% de los participantes pertenece a Q1 y Q2, en la del Bío-Bío sube al 50\%, y luego baja al 44\% en la de Los Lagos para, finalmente, en la Metropolitana, llegar al 32\%. Este hecho también va acompañado de una variabilidad de los participantes de Q5. En la de Valparaíso es de $11 \%$, en la del Bío-Bío de 5,3\%, del 15\% en la de Los Lagos y del $18 \%$ en la Metropolitana, evidenciando la complejidad de esta relación, mediatizada por oportunidades de instituciones de ES muy diversas, recursos económicos, entre otros.

\subsection{Los estudiantes de los institutos profesionales (IP)}

En la muestra participaron 1.479 estudiantes de IP. Un rasgo distintivo es que disminuye su edad -se reducen los alumnos de más de 31 años-aumentando correlativamente los estudiantes de "dedicación exclusiva" (77\%). La segunda relación es que, a mayor edad, se pertenece a un quintil superior, valor con significación estadística ( $\mathrm{Chi}^{2}$ de 53,3***4/16 gl). También, ello se ha traducido en una reducción de 3\% del grupo que trabaja. De igual forma, han disminuido a la mitad los estudiantes pobres no indigentes (5\%), representando el total de no pobres al 93\% de estudiantes de IP. El conjunto de cifras ratifica el desplazamiento mayor de este grupo hacia la dedicación exclusiva al estudio.

Una segunda área de diferencias con los CFT corresponde a la estructura por género del estudiantado. En los IP los alumnos son esencialmente hombres solteros ( $\mathrm{Chi}^{2}$ de $12,73 * * * 4 / 1 \mathrm{gl}$ ), evidenciando que no es una situación fortuita. Las mujeres conforman

8 Se trata de una limitación de la base de datos. 
un grupo pequeño, predominando el estado civil "separada", presentando además una situación de más vulnerabilidad al configurar, mayoritariamente el grupos de indigentes y el de pobres (Chi ${ }^{2}$ 10,946 *** (2/l gl). Las mujeres son dominantes en los grupos de indigentes y pobres, presentando mayor vulnerabilidad. En el caso de los hombres, ambas categorías reúnen al 4,9\% de los estudiantes, en las mujeres se eleva al 8,8\%. De cada tres pobres o indigentes dos son mujeres.

En materia de trabajo, los de Q1 tienen menos trabajo (1\% total), igual que en los CFT. En el análisis por género, seis de cada 10 estudiantes que trabajan son hombres. Ninguno de los 29 estudiantes indigentes tenía trabajo y, del total de estudiantes que trabaja, el 92\% son "no pobres". Aquellos que han buscado trabajo son aproximadamente un 3\% del total. No hay tendencias por género, mientras que por situación de pobreza es consistente con la estructura de quintiles. Las mujeres no buscan trabajo debido a su dedicación a labores del hogar y/o al cuidado de hijos y de la familia, aspecto transversal a los quintiles y a los niveles (CFT, IP, UES). De igual forma, el análisis por situación de pobreza no da más luces a lo expuesto.

La mayor parte de quienes trabajan lo hacen como empleados $\mathrm{u}$ obreros, sea en el sector privado o -en menor medida- en el público (dos a uno). Los empleos del sector privado son de mayoría masculina; los del público, femenino. La situación laboral les diferencia del grupo de CFT. El 74\% tiene empleo permanente, pero sin dominio por quintil. Según género trabajan un $11 \%$ más de hombres. La pertenencia a sistemas previsionales alcanza al $65 \%$ de quienes trabajan, con un fuerte dominio de los hombres no pobres.

Tabla 12. Trabajo según quintil-IP

\begin{tabular}{|c|c|c|c|c|c|c|c|}
\hline & \multicolumn{5}{|c|}{ Quintil } & \multirow{2}{*}{ Total } \\
\hline & & 1 & 2 & 3 & 4 & 5 & \\
\hline \multirow[t]{2}{*}{ Sí } & Casos & 15 & 54 & 71 & 110 & 104 & 354 \\
\hline & $\%$ quintil & $9,9 \%$ & $20,0 \%$ & $21,4 \%$ & $26,8 \%$ & $33,1 \%$ & $23,9 \%$ \\
\hline \multirow{3}{*}{ No } & $\%$ total & $1,0 \%$ & $3,7 \%$ & $4,8 \%$ & $7,4 \%$ & $7,0 \%$ & $23,9 \%$ \\
\hline & Casos & 137 & 216 & 261 & 301 & 210 & 1.125 \\
\hline & $\%$ quintil & $90,1 \%$ & $80,0 \%$ & $78,6 \%$ & $73,2 \%$ & $66,9 \%$ & $76,1 \%$ \\
\hline
\end{tabular}




\begin{tabular}{c|l|c|c|c|c|c|c}
\hline \multirow{3}{*}{ Total } & $\%$ total & $9,3 \%$ & $14,6 \%$ & $17,6 \%$ & $20,4 \%$ & $14,2 \%$ & $76,1 \%$ \\
\cline { 2 - 8 } & Casos & 152 & 270 & 332 & 411 & 314 & 1.479 \\
\cline { 2 - 8 } & $\%$ itrabajó? & $10,3 \%$ & $18,3 \%$ & $22,4 \%$ & $27,8 \%$ & $21,2 \%$ & $100,0 \%$ \\
\hline \multirow{2}{*}{} & $\%$ quintil & $100,0 \%$ & $100,0 \%$ & $100,0 \%$ & $100,0 \%$ & $100,0 \%$ & $100,0 \%$ \\
\hline
\end{tabular}

Complementariamente, el Q1 es el grupo que tiene menos trabajo (-10\%). En Q2 y Q3 se duplica la participación relativa del primero (20\% y más), con una representación sobre el total de la población del 3,7 al 4,8\%, dando cuenta de un bajo porcentaje aún. En Q4 trabaja el 26\% y en Q5 el 33\%; lo cierto es que su peso sobre el grupo total es similar, 7\%, aproximadamente, tampoco de gran significación. De esta forma, en la medida en que se identifica un sujeto de un quintil superior, de manera concomitante aumenta el porcentaje de los que trabajan, como igualmente aumenta su edad y, por ende, debería asociarse a la búsqueda del autosustento.

Una pregunta importante para los CFT e IP es determinar si la situación laboral es una condición previa, simultánea o posterior a su incorporación a la institución. De ser una característica previa, su comportamiento estaría asociado a su decisión de estudiar -por ende, es una condición asumida- e introduciría menos riesgos sobre el término exitoso de los estudios que si fuese una característica generada cuando la persona ingresa a estudiar, sea por un requerimiento asociado al financiamiento de sus estudios o que provenga de otra exigencia, el hecho que haya decidido trabajar después de la decisión de estudiar incrementa el riesgo de una menor retención.

Dado el bajo interés en buscar trabajo que muestran los estudiantes y que su distribución no tiene sesgo hacia los primeros quintiles, hay que identificar el trasfondo que explica la falta de resultados de esa búsqueda y su eventual anomia o, bien, su asociación con el capital social y cultural del núcleo familiar. De manera que si bien uno de cada cinco estudiantes trabaja, en los cuatro restantes parece estar internalizada la condición de que "su trabajo es estudiar". Segundo, los indigentes no tienen trabajo y muchos de los pobres tampoco. De igual manera, un grupo menor de ellos ha buscado realmente trabajo, asumiendo que es inevitable no tenerlo, 
independiente de la estructura de necesidades (o bien la estructura de necesidades incide de manera diferente a lo estimado).

Ya que la cobertura de becas y ayudas no es alta, sino, por el contrario, más bien reducida, y no siempre son asignadas a los estudiantes de los primeros quintiles, hay brechas de satisfacción de necesidades que sería interesante estudiar con mayor detenimiento.

Ajeno a las condiciones socioeconómicas y de género, hay estudiantes de IP que no tienen acceso a equipamientos relevantes para su calidad de vida en el hogar, situación que demanda un análisis más detallado del tema. De igual forma, el acceso a computador es en ellos un factor clave y está sesgado negativamente hacia los primeros dos quintiles. El uso del computador sigue un patrón independiente de las consideraciones de género. Su predominio es para estudio y otro tanto para trabajo, y lo comparten con los alumnos de CFT y de las UES. Situación análoga se da en el uso de Internet: se emplea para la obtención de información, sin distingos por género.

Tabla 13. Acceso a computador por quintil-IP.

\begin{tabular}{|c|c|c|c|c|c|c|c|}
\hline \multirow{2}{*}{\multicolumn{2}{|c|}{$\begin{array}{l}\text { Acceso a } \\
\text { computador }\end{array}$}} & \multicolumn{5}{|c|}{ Quintil } & \multirow{3}{*}{$\begin{array}{l}\text { Total } \\
1.322\end{array}$} \\
\hline & & \multirow{2}{*}{$\begin{array}{c}1 \\
123\end{array}$} & \multirow{2}{*}{$\begin{array}{c}2 \\
222\end{array}$} & \multirow{2}{*}{$\begin{array}{c}3 \\
288 \\
\end{array}$} & \multirow{2}{*}{$\begin{array}{c}4 \\
387 \\
\end{array}$} & \multirow{2}{*}{$\begin{array}{c}5 \\
302 \\
\end{array}$} & \\
\hline Sí & Casos & & & & & & \\
\hline & $\%$ acceso & $9,3 \%$ & $16,8 \%$ & $21,8 \%$ & $29,3 \%$ & $22,8 \%$ & $100,0 \%$ \\
\hline & $\%$ quintil & $80,9 \%$ & $82,2 \%$ & $86,7 \%$ & $94,2 \%$ & $96,2 \%$ & $89,4 \%$ \\
\hline \multirow{4}{*}{ No } & $\%$ total & $8,3 \%$ & $15,0 \%$ & $19,5 \%$ & $26,2 \%$ & $20,4 \%$ & $89,4 \%$ \\
\hline & Casos & 29 & 48 & 44 & 24 & 12 & 157 \\
\hline & $\%$ acceso & $18,6 \%$ & $30,1 \%$ & $28,2 \%$ & $15,4 \%$ & $7,7 \%$ & $100,0 \%$ \\
\hline & $\%$ quintil & $19,1 \%$ & $17,4 \%$ & $13,3 \%$ & $5,8 \%$ & $3,8 \%$ & $10,5 \%$ \\
\hline \multirow{4}{*}{ Total } & $\%$ total & $2,0 \%$ & $3,3 \%$ & $3,0 \%$ & $1,6 \%$ &, $8 \%$ & $10,6 \%$ \\
\hline & Casos & 152 & 270 & 332 & 411 & 314 & 1.479 \\
\hline & $\%$ acceso & $10,3 \%$ & $18,3 \%$ & $22,4 \%$ & $27,8 \%$ & $21,2 \%$ & $100,0 \%$ \\
\hline & $\%$ quintil & $100,0 \%$ & $100,0 \%$ & $100,0 \%$ & $100,0 \%$ & $100,0 \%$ & $100,0 \%$ \\
\hline
\end{tabular}

La consideración final proviene del análisis de los resultados por género. La disponibilidad de computador es favorable tres a uno a los hombres. Claramente, la condición de género limita esta posibilidad. Los datos ameritan que las instituciones revisen con 
mayor detenimiento esta situación, dadas las implicancias que tiene para la retención de sus estudiantes.

\subsection{Los estudiantes de las universidades públicas y privadas}

Los estudiantes de las UES públicas son 2.857 equivalente al 65,4\% del total, los de UES privadas (UP) registran 1.514 alumnos, representando el 34,6\%. La creciente concentración de la matrícula del sistema de ES en este segmento y la orientación que adquiere la mayor parte de los instrumentos de apoyo social y económico para dar soporte a los estudiantes, hacen del sistema universitario un componente estratégico de la ES y del desarrollo del país.

Como fue señalado, los estudiantes universitarios conforman la gran mayoría de los alumnos de la ES, por ello, desde esta perspectiva, el análisis de sus características genera un impacto agregado mayor. También se sabe que cualquiera sea el quintil de pertenencia, el género, la situación de pobreza y de edad, cuantitativamente el mayor número de casos corresponde al sistema universitario.

Aun con esta homogeneidad, existen diferencias relevantes. Primero, las UES públicas acogen en mayor medida a los estudiantes de los primeros tres quintiles de la población. Correspondiendo casi a la mitad en las UES Cruch (49\%) y menos de un tercio a las privadas (30\%). Esta situación se equilibra en Q4, mientras que el grupo de estudiantes de Q5 está dominado por las UP que, porcentualmente, les supera por 20 puntos $\left(\mathrm{Chi}^{2} 210,801^{* * *}\right)$.

Tabla 14. Quintil según dependencia de la universidad (2003)

\begin{tabular}{l|l|c|c|c}
\hline \multirow{2}{*}{ Quintil } & \multicolumn{2}{|c}{ Dependencia Universidad } & \multirow{2}{*}{ Total } \\
\cline { 3 - 4 } \multicolumn{2}{l|}{1} & Cruch & Privada & \\
\hline \multirow{3}{*}{2} & $\%$ dependencia & 302 & 91 & 393 \\
\hline \multirow{3}{*}{2} & $\%$ total & $10,6 \%$ & $6,0 \%$ & $9,0 \%$ \\
\cline { 2 - 4 } & Casos & $6,9 \%$ & $2,1 \%$ & $9,0 \%$ \\
\cline { 2 - 4 } & $\%$ dependencia & 478 & 136 & 614 \\
\hline
\end{tabular}




\begin{tabular}{c|l|c|c|c}
\hline \multirow{3}{*}{3} & $\%$ total & $10,9 \%$ & $3,1 \%$ & $14,1 \%$ \\
\cline { 2 - 5 } & Casos & 617 & 228 & 845 \\
\cline { 2 - 5 } & $\%$ dependencia & $21,6 \%$ & $15,1 \%$ & $19,3 \%$ \\
\hline \multirow{4}{*}{4} & $\%$ total & $14,1 \%$ & $5,2 \%$ & $19,3 \%$ \\
\cline { 2 - 5 } & Casos & 728 & 366 & 1.094 \\
\cline { 2 - 5 } & $\%$ dependencia & $25,5 \%$ & $24,2 \%$ & $25,0 \%$ \\
\hline \multirow{3}{*}{5} & $\%$ total & $16,7 \%$ & $8,4 \%$ & $25,0 \%$ \\
\cline { 2 - 5 } & Casos & 731 & 692 & 1.423 \\
\cline { 2 - 5 } & $\%$ dependencia & $25,6 \%$ & $45,7 \%$ & $32,6 \%$ \\
\hline \multirow{3}{*}{ Total } & $\%$ total & $16,7 \%$ & $15,8 \%$ & $32,6 \%$ \\
\cline { 2 - 5 } & Casos & 2856 & 1513 & 4.369 \\
\cline { 2 - 5 } & $\%$ quintil & $65,4 \%$ & $34,6 \%$ & $100,0 \%$ \\
\hline & $\%$ dependencia & $100,0 \%$ & $100,0 \%$ & $100,0 \%$ \\
\hline
\end{tabular}

El análisis según condición de pobreza muestra una relación consistente con diferencias menores con los CFT e IP. Los estudiantes indigentes se reducen del 2 al 1,3\% del total, identificándose 3,1 estudiantes en UES Cruch, por 1 en UP. El grupo de alumnos pobres se incrementa respecto de los IP llegando al 5,6\% del total. Sumados ambos mantienen su peso total, aunque cambia su composición. Aumentan los pobres pero se mantiene el registro proporcional de no pobres (93\%) que constituyen la mayoría de los estudiantes universitarios, igual en los IP, y semejante a la serie anterior de años. Los alumnos pobres también se inclinan por las UES Cruch en una relación dos a uno respecto de las UP.

Las becas de sustento tienen sesgo positivo hacia los primeros quintiles, con más fuerza en UES públicas (13,3\%) que en UP $(2,1 \%)$, siendo las becas de colegiatura en ambos casos más numerosas. En las públicas corresponden al 21,4\% (las estatales son el 75\% del total). Las becas Presidente de la República (PR) están asignadas en grado importante con afirmación positiva en estudiantes de Q1 a Q3. En las UP las becas PR siguen lo descrito, aunque con una cobertura menor (1,9\%). En el caso de las otras becas estatales (1,9\%) la tendencia es igual. Las becas no estatales en las UES públicas se concentran en los últimos dos quintiles (+2\%) pero, de todas formas, en éstas se reducen significativamente las diferencias entre los primeros tres quintiles con los dos superiores $\left(\mathrm{Chi}^{2}\right.$-aj- de 24,1***). 
Específicamente, un 4,3\% de los estudiantes pertenece a alguna etnia o pueblo indígena, lo que predomina en las UES públicas. Aunque inicialmente ello se asociaría a su condición socioeconómica, los datos no lo ratifican. Un tercio es objeto de becas de colegiatura en las UES públicas y 1/5 en las UP, no cubriendo a todos los estudiantes pobres e indigentes.

Respecto del estado civil, se mantiene lo preestablecido. La mayor parte de los estudiantes son solteros (91\%). El grupo de casados/convivientes es reducido (9\%), asociado a los quintiles y edades superiores, sin destacar diferencias de significación. Los separados son una fracción menor a 0,5\%. En el plano de tendencias, hay más mujeres casadas y separadas que hombres.

Por género, en las UES públicas no hay diferencias. En las UP el 55\% de su alumnado es mujer (Chi2 9,977**). Otros antecedentes en esta línea muestran que la variable edad no tiene incidencia significativa, como podría tener la localización geográfica de los estudiantes, ya que en el caso de las UP el 50\% pertenece a la Región Metropolitana, lo que en las públicas es del 23\% (en las UP 1 de 2 es de la RM, en las públicas 1 de 4).

En materia de empleo, el 18\% de los alumnos de las UES públicas trabaja y un 23\% de las UP. El grupo de Q4 es, proporcionalmente, el que más trabaja, lo que se podría explicar porque, comparativamente, recibe menos beneficios (crédito y becas) que el de los primeros quintiles (en promedio un 64\% de los alumnos tiene 60\% o más de crédito universitario), mientras que recibe casi la misma cobertura que Q5 (poco más del 50\% de los alumnos de Q4 y Q5 reciben sobre el 60\% de crédito), correspondiendo a un segmento de menores ingresos.

Respecto del precio de las carreras, en las UP se destaca que tanto Q1 como Q5 tengan al menos el 50\% de sus estudiantes pagando los valores más elevados, lo que en las UES Cruch responde a menos de la mitad en Q1 y a 17 puntos menos en Q5. Es una relación 
estadísticamente significativa para las UES públicas y no para las UP. Por género se presenta la misma significación estadística para las públicas: en los tres quintiles bajos predominan las mujeres, en los dos más altos los hombres.

Profundizando el tema precio mensual de la carrera (pago mensualidad), la situación presenta algunas complejidades. La mayor parte de los alumnos se encuentra estudiando carreras de $\$ 120.000$ mensuales ${ }^{9}$ y más ( $55 \%$ en las UES Cruch y $65 \%$ en las UP), de forma que el costo de la carrera siendo un dato importante, pero no es el único que se asume para esta decisión. Por otra parte, hay un racional más fuerte en los estudiantes de las UES públicas, ya que de Q1 a Q3 el $26 \%$ aproximado cursa carreras de menos $\$ 100.000$ mensuales, cifra que desciende a 21\% en Q4 y 17\% en Q5. Ello acontece en forma parecida en las UES privadas ${ }^{10}$. Sin embargo, en estas últimas hay una fuerte concentración de los estudiantes en las carreras más costosas, ello acontece en todos los quintiles y con una diferencia muy superior (cuando ello ocurre) respecto de lo que se registra en las UES Cruch.

En materia de crédito, los alumnos de los quintiles inferiores asumen mayor compromiso financiero por coberturas mayores. Alternativamente, para los tramos superiores de crédito los valores se invierten.

\section{Cambios en el acceso a la ES: debates pendientes}

La ES chilena ha mutado significativamente el último cuarto de siglo. Nuevos actores institucionales, programas académicos, modalidades de trabajo y, esencialmente, una masiva incorporación de nuevos estudiantes de diversos orígenes dan cuenta de una realidad que hay

9 Precio en pesos del año 2003.

10 Podría ocurrir que los estudiantes de los primeros quintiles, como grupo, tengan resultados en la enseñanza secundaria más bajos y se inserten en carreras de menor precio, que son menos demandantes de puntajes de selección más elevados. Aunque sabemos que este proceso se encuentra predeterminado en grado importante por la educación de los padres, la que también tiene sesgos significativos hacia los estudiantes de los primeros quintiles (Donoso, 2003). 
que considerar. El concepto de estudiante con dedicación exclusiva está siendo incipientemente compartido con el de estudiante de dedicación parcial.

\subsection{Estratificación institucional de la ES}

La ES experimenta un proceso de estratificación socioinstitucional importante. El segmento universitario sin contrapeso relevante reúne la mayor demanda absoluta por matrícula en todos los quintiles, edades y géneros. Sin embargo, referido a valores relativos hay tendencias institucionales: los CFT atienden a la población más pobre o perteneciente a los primeros quintiles. También reúne a los dos extremos etarios: tiene los alumnos más jóvenes como también al grupo de mayor edad. Adicionalmente, las mujeres integran su estudiantado dominante de los primeros quintiles, siendo además el segmento institucional que tiene la mayor cantidad de estudiantes con trabajo remunerado.

Los IP constituyen una realidad diferente. En primera instancia, sus estudiantes provienen masivamente de los primeros cuatro quintiles, teniendo también participación menor (relativa) Q5. Proporcionalmente cuentan con mayor población joven. Por género, hay un marcado predominio masculino. Trabajan en menor cantidad que el grupo anterior. Si bien son razonables, en sus estándares de calidad de vida se aprecia postergación del bienestar presente por invertir en educación.

Las UES públicas concentran la mayor población de todos los estratos, niveles, géneros y edades, representando la diversidad país. Hay distribución equivalente por género y grupos de edad. Contribuye a esta situación el soporte de becas de manutención y matrícula que poseen. La mayor cantidad de estudiantes más pobres asiste a estos planteles. Los estudiantes que trabajan se reducen a uno de cada cinco.

Las UES privadas reúnen la mayor participación relativa de estudiantes del Q5 y también de mujeres. También cuentan con 
estudiantes de los primeros quintiles, pero en una proporción significativamente menor que las UES públicas. Son similares etariamente en términos de población y muestran una relación próxima a la UES públicas en materia laboral.

\subsection{Estratificación social: relación estudiantes instituciones}

Los más pobres no llegan masivamente a la ES. Sí hay estudiantes indigentes y pobres, pero los "no pobres" son la figura dominante del sistema en todos sus niveles (93\%). El grupo de mayor vulnerabilidad lo conforman las mujeres. Esta situación es importante actualmente por su incorporación masiva y creciente al sistema. Debe agregarse que las mujeres cuentan con menos soporte familiar para estudiar, mayores dificultades de inserción laboral, más responsabilidades sociales y familiares, y menor cobertura de sistemas previsionales, siendo las principales demandantes de salud pública en todos los quintiles.

Las becas de soporte y matrícula tienen comportamientos diferentes según la institución de ES. Aunque se puede sostener que son asignadas por afirmación positiva, hay diferencias importantes en esta materia, una de las cuales se vincula con la población de origen indígena.

La brecha digital es una materia con cierto sesgo socioeconómico que no discrimina por tipo de institución, observándose diferencias importantes (15 puntos porcentuales) de cobertura entre los quintiles 1 y 5 en todas las instituciones, salvo en las UES públicas, en las cuales la diferencia cae a la mitad; como también -en oportunidades- por género, perjudicando a las mujeres. Sumados los aspectos de calidad de vida de la población, con el soporte requerido para estudiar, muestran que los estudiantes indigentes, pobres y una fracción relevante de los no pobres (equivalente a Q2, Q3 y Q4) postergan en parte su mejor calidad de vida por atender el financiamiento de sus carreras. Ello se traduce en mayor endeudamiento. Esta situación responde a la gran esperanza depositada en la educación para gestar cambios positivos, como también de movilidad social ascendente a 
la que aspira una gran parte de la sociedad. Posiblemente por ello los quintiles con menor capital social y económico no son los que privilegian el trabajo mientras estudian. El costo/oportunidad en este grupo es mayor (se incrementa aún más en las mujeres) y lo rentabilizan centrándose en el estudio.

\subsection{Desafíos pendientes}

El desarrollo de la ES implica crecimiento y calidad. Compatibilizar estos considerados con incremento de la calidad de la formación y de la retención de estudiantes constituye un reto mayor. Hay cuestiones no menores, como la flexibilidad institucional para adaptarse a los nuevos tipos de alumnos, sobre las que el mercado deberá operar a la brevedad.

La extraordinaria demanda por ES exige seriedad y responsabilidad de las instituciones hacia sus estudiantes. Los alumnos de los primeros quintiles requieren mayor soporte académico, específicamente en materia digital y en pautas de funcionamiento en la "sociedad de la ES". Paralelamente, la incorporación de población más pobre feminiza aún más la ES, implicando soporte agregado en otras materias: tiempos de funcionamiento, atención de salud, por ejemplo, lo cual implica ascender a una escala de políticas país.

La provisión de las oportunidades educacionales en los momentos adecuados se confirma en los análisis realizados: la "sobreedad" conlleva mayores obstáculos para estudiar y presenta más dificultades para permanecer (retención). Puede suplirse con mayor capital económico, pero ello discrimina negativamente a pobres e indigentes (primeros quintiles) y, además, afecta en mayor medida a las mujeres.

La temática de los estudiantes de ES que trabajan requiere mayores antecedentes y un estudio acabado. Tras este fenómeno hay temas culturales asociados al hábito del trabajo, comportamientos de género, prácticas de responsabilidad personal, social y familiar; además, elementos asociados al capital social y cultural que superan las 
necesidades específicas de financiamiento de los estudiantes, y abren interrogantes para identificar líneas de acción que las instituciones de ES, con visión prospectiva, incorporen en sus políticas de desarrollo de mediano y largo plazo.

A partir de lo expuesto, se hace evidente una política (sin desmedro de que las instituciones ya las posean) de apoyo a los estudiantes más vulnerables, que contribuya a una mayor equidad en términos de resultados educativos y que se oriente a quienes pertenecen a los primeros tres quintiles, esencialmente a las mujeres, iniciativa que, adicionalmente, debe tener arraigos importantes en las diferentes características de los estudiantes según su nivel institucional: CFT, IP, UES públicas y UES privadas.

Por ende, se hace indispensable articular las políticas sectoriales -salud, telecomunicaciones, entre otras-con visiones territoriales que generen mejores condiciones de educabilidad, para que la población de menores recursos -mayoritariamente mujeres- potencien sus oportunidades.

La educación superior es un sector clave de y para el desarrollo del país. El proceso de "universitarización" creciente no se condice con todos los discursos en este campo. Pero el estancamiento de la demanda y la estigmatización de las instituciones no universitarias dan origen a fenómenos de exclusión social que debiésemos evitar. La complejidad de las tareas del sector requiere de políticas sustentables basadas en la construcción de alianzas de largo plazo entre Estado y privados, y no en la exacerbación de fundamentalismos maniqueístas que buscan dirimir entre Estado y mercado.

La confianza de la sociedad se expresa en la correspondencia entre las oportunidades reales y las ilusiones. La retención de los estudiantes -basada en logros de aprendizaje-es una tarea que supera las políticas de financiamiento, las cuales, siendo necesarias, no son suficientes para provocar que la población con mayores carencias supere los obstáculos de la formación superior. En este proceso, el 
soporte de las opciones de formación en estrategias de aprendizaje, herramientas tecnológicas, por ejemplo, es un factor clave en un área que debe recuperar como finalidad la visión de mediano y largo plazo en la inversión en capital humano.

Agradecemos el apoyo financiero del Consejo Superior de Educación (CSE). Hacemos extensivo esta consideración al Departamento de Didáctica y Organización Escolar de la Universidad de Valencia, como también a la Universidad de Talca y a la Organización de Estados Iberoamericanos para la Educación, la Ciencia y la Cultura (OEI), Chile.

\section{Referencias bibliográficas}

Bean, J. (1980) Dropouts and turnover. The synthesis of a test of causal models of student attrition. Research in Higher Education, 12, pp. 155-187.

Bean, J. (1983) The application of model of turnover in work organizations to the student attrition process. Review of Higher Education, 6 (2), pp. 129-148.

Bean, J. (1985) Interaction effects based on class level in an explanatory model of college student dropout syndrome. American Educational Research Journal, 22 (1), pp. 35-64.

Bean, J. y Vesper, N. (1990) Quantitative Approaches to Grounding Theory in Data: Using LISREL to Develop a Local Model and Theory of Student Attrition. Boston: EU, Annual Meeting AERA.

CSE (2006) INDICES 2006. Santiago de Chile: Consejo Superior de Educación. www.ces.cl (Consultado octubre 2006)

Donoso, S. (2003) Éxito y fracaso de los participantes en las etapas del proceso de selección a las universidades del Consejo de Rectores. Variables Asociadas. Santiago de Chile: Universidad Academia de Humanismo Cristiano.

Dooner, C. (2005) Expansión de las universidades e Institutos profesionales cuya autonomía ha sido certificada por el CSE. Calidad en la Educación, 22, julio, pp. 175-232.

Ethington, C. A. (1990) A psychological model of student persistence. Research in Higher Education, 31 (3), pp. 266-269. 
Fishbein, M. \& Ajzen, I. (1975) Belief, attitude, intention and behavior: An Introduction to theory and Research. MA, EE.UU.: Addison- Wesley, Reading.

González, L.E. y D. Uribe (2002) Estimaciones sobre la repitencia y deserción en la educación superior chilena. Consideraciones sobre sus implicancias. Calidad de la Educación, $2^{\circ}$ semestre pp. 75 -90.

González, P. (2005) La igualdad educativa, el financiamiento vía subvenciones y la administración privada de la educación: elementos para un debate. García Huidobro, J. E. (Ed) Políticas Educativas y Equidad. Fundación Ford, UNESCO UNICEF, Santiago de Chile, pp. 247 -270.

Himmel, E. (2002) Modelos de análisis para la deserción estudiantil en la educación superior. Calidad de la Educación, 17, diciembre, pp. 91-107.

Illanes, M. I. (1991) Ausente señorita: el niño chileno y la escuela para pobres y el auxilio 1890 -1990. Santiago de Chile: JUNAEB.

INE (2004) Estimaciones de la demanda por educación.

Leiva, A (2005) Ejes para la elaboración de una política a la educación superior. Calidad de la Educación, 22, julio, pp. 37-68.

MIDEPLAN (2003) Escuesta Casen, www.mideplan.cl/casen años 2003.

MINEDUC (1964) Algunos Antecedentes para el Planeamiento Integral de la Educación Chilena. Santiago de Chile: Ministerio de Educación, Editorial Universitaria.

MINEDUC (2002) Estadísticas anuales. Santiago de Chile: Ministerio de Educación.

Pascarella, E. T., Smart, J. C. \& Ethington, C. A. (1986) Long-term persistence of two-year college students. Research in Higher Education, 24 (1), pp. 47-71.

Pascarella, E. \& Terenzini, P. (1991) How college affects students. San Francisco: Jossey- Bass.

Pascarella, E. T. \& Terenzini, P. T. (1980) Predicting freshmen persistence and voluntary dropouts decisions from a theoretical model. Journal of Higher Education, 51 (1), pp. 60-75.

PNUD, Mideplan (2005) Expansión de la Educación Superior en Chile. Hacia un Nuevo enfoque de la Calidad y Equidad. Santiago de Chile.

Robbins, S., Lauver, K., Le, H., et al. (2004) Do psychological and study skill factors predict college outcomes? A Meta-Analysis. Psychological Bulletin, 130(2), pp. 261-288. 
Rodríguez, C., Donoso G. y Zunino, E. (1982) Deserción y cambio de carrera en la Universidad de Chile. Santiago de Chile: División Estudios, Servicio de Selección y Registro de Estudiantes, Universidad de Chile, Monografía $\mathrm{N}^{\circ} 14$.

Schiefelbein, E. (1975) Repeating: an overlooked problem of Latin American Education. Comparative Education Review, (19), pp. 468 -487.

Schiefelbein, E. (1976) Diagnóstico del sistema educacional chileno en 1970. Santiago de Chile: Universidad de Chile, Departamento de Economía.

Schiefelbein, E. \& Simmons J. (1981) The determinants of school achievement. The review of the research for developing countries. Ottawa: IDRC.

Schiefelbein, E. \& Farell, J. (1982) Eight years of their lives: Through schooling to the labour market in Chile. Ottawa: IDRC.

Spady, W. G. (1970) Dropouts from higher education: An interdisciplinary review and synthesis. Interchange, 1(1), pp. 64-85.

Stevens, G. (2007) Exclusión o autoexclusión: Caracterización de aquellos que se inscriben y no rinde la PSU. Tesis para optar al Grado Académico de Magíster en Política y Gestión Educacional. Universidad de Talca, Instituto de Investigación y Desarrollo Educacional. (Proyecto Fondecyt 1051015).

Tinto, Vincent (1975) Dropout from Higher Education: A theoretical synthesis of recent research. Review of Educational Research, 45: pp. 89-125.

Tinto, V. (1982) Limits of theory and practice of student attrition. Journal of Higher Education, 53(6), pp. 687-700.

Tinto, Vincent (1986) Theories of student departure revisited. In D. Smart (ed.) Higher Education Handbook of Theory and Research (Vol. 2) New York: Agathon Press.

Tinto, Vincent (1987) Leaving College. Chicago: The University of Chicago Press.

Tinto, Vincent (1997) Classrooms as communities: Exploring the educational character of student experience. Journal of Higher Education, 68(6), pp. 599-623.

Tinto, Vincent (1998) Colleges as communities: taking research on student persistence seriously. The educational character of student experience. Review of Higher Education, 21(2), pp. 167-177. 
244 CARACTERIZACIÓN SOCIOECONÓMICA - Sebastián Donoso, Víctor Cancino

Weidman, J. (1989) Undergraduate socialization: a conceptual approach. En: J. Smart (ed.) Higher education: Handbook of theory and research (Vol. 5). New York: Agathon.

Recibido: 16 abril de 2007 Aceptado: 3 de mayo de 2007 